Notes :

1. Voir bibliographie en fin d'article

2. Outre les travaux cités dans la bibliographie, une conférence donnée par A. Hasnaoui à l'Institut du Monde Arabe à Paris en décembre 2005 a largement inspiré une partie de cet exposé.

3. Noté JP par la suite

4. Le qualificatif souvent utilisé en philosophie des sciences de néoplatonicien, et souvent attribué à Philopon, nous semble en général, tout comme dans son cas particulier, inadapté et peu fécond pour l'analyse.

5. Plusieurs auteurs affirment que ce concept était déjà présent dans les thèses de Hipparque de Nicée, $\Pi^{e}$ siècle avant JC.

6. Noté $\mathrm{AB}$ dans la suite

\title{
Bibliographie succincte
}

1. Aristote, Physique. Traduction de Pierre Pellegrin, GF Flammarion, 2000.

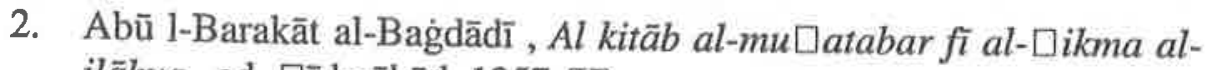
ilāhya, ed. 口īderābãd, 1357 (H)

3. Gutas D., Pensée grecque, culture arabe, ed. Aubier 2005, première édition Greek Thought, Arabic Culture, Routledge, 1998

4. Hasnaoui A., Le statut catégorial du mouvement chez Avicenne, in Cahiers du Mideo, 2004, La définition du mouvement dans la Physique du Shifa' d'Avicenne, in Arabic Sciences and Philosophy 11, 2001.

5. Pinès S., Studies in Abū l-Barakāt al-Bağdādī Physics and Metaphysics, in Collected Works of Shlomo Pines, The Hebrew University, Jerusalem, 1979

6. Sorabji R., John Philoponus. In: Philoponus and the Rejection of Aristolelian Science. Duckworth, London, 1991. 
- Quelle a été la postérité d'Abū 1-Barakāt dans le monde musulman ? Dans le monde latin ? Est-il possible que son apport, notamment sur le lien entre force et accélération, ait alimenté les avancées du XVIè et du XVIIè ? Sous quelle forme ? Il n'y a pas le début d'une réponse à ces questions dans le domaine de la physique du mouvement : le terrain est vierge puisque la question a été longtemps déclarée comme nulle et non avenue.

\section{Conclusion}

Il est saisissant quand on fait de l'histoire et de la philosophie des sciences, de voir à quel point de petites questions qui semblent «techniques », peuvent faire basculer la représentation du monde de toute une société. $\mathrm{Si}$ on redonnait à la physique cette dimension de profond engagement dans la pensée humaine, le gain serait considérable pour les jeunes d'une part, et pour la science d'autre part.

Le Doyen de la faculté des Arts, Dr Ahmed Abdallah Zayed, évoquait ce matin en introduction le rôle que devrait jouer la culture classique pour lutter contre la violence et les extrémismes.

Pour ma part, je ne suis pas sûre que la culture classique soit, par nature, un antidote aux extrémismes. Pour ne prendre qu'un seul exemple, les officiers nazis étaient en majorité des gens très cultivés et bon nombre d'entre eux connaissaient à fond «leurs classiques», notamment grecs. C'est davantage l'aventure humaine et le mouvement des idées au sein des cultures passées ou contemporaines, dont l'analyse peut favoriser cela. A condition d'éviter deux écueils : une lecture identitaire bien sûr, mais aussi une lecture ocuménique qui amènerait à conclure que tout est dans tout et qu'aujourd'hui est la continuité d'hier. Il s'agit donc de lire dans la rencontre des cultures, «toutes hybrides » disaient E.W. Saïd, mais aussi «extraordinairement différenciées » ce qui a pu amener à construire des ruptures épistémologiques fécondes. Le geste créateur des physiciensphilosophes dont il est question ici, emprunte peut-être plus souvent aux lisières entre ces cultures qu'à leur identité... 


\subsection{L'impetus appliqué à l'explication de « l'accélération » de la chute des graves}

Aristote n'a jamais proposé d'explication à l'accélération de la vitesse des graves. Ibn Sīnā est le premier à appliquer la notion d'impetus pour expliquer cette modification de la vitesse. Il établit un parallèle entre une puissance qualitative et l'action de la gravité. De même qu'une matière qui a déjà en elle un certain degré de chaleur est plus apte à recevoir une chaleur plus intense, de la même manière un corps animé d'un mouvement naturel qui a déjà un impetus engendré en lui par la gravité est plus apte à recevoir un impetus plus intense.

Abū l-Barakāt reprend et affine cette analyse en reliant explicitement l'accélération à la notion de force, qui était jusque là systématiquement reliée à la notion de vitesse. C'est là un apport très important à la science du mouvement et il nous reste à chercher les prolongements dans l'histoire de cette science qu'une telle avancée a pu permettre. Sa conception du vide est sans doute en rapport avec le mu'atazilisme, héritier d'un certain atomisme qui n'est pas celui de Lucrèce (inconnu des arabes) mais peut être celui de Démocrite et Épicure, connu à travers les commentaires des philosophes grecs. C'est avec Abū l-Barakāt que la conception du lieu comme espace tridimensionnel est poussée pour la première fois jusqu'au bout : jusqu'à en déduire que l'espace est infini.

\subsection{Transmission et postérité}

- On sait maintenant avec beaucoup de détails comment le texte de la physique d'Aristote et ses commentaires comme celui de JP (pas tous) ont été connus des arabes. On sait moins le chemin des écoles philosophiques et de nombreux débats sur les questions d'héritage (plus ou moins féconds) ont lieu. Les philosophes arabes aspiraient euxmêmes fortement à se présenter comme les dépositaires et héritiers du savoir grec. 
et simplement celui d'AB.

Or Abū l-Barakāt ${ }^{(6)}$ reprend et prolonge de façon audacieuse le commentaire de Philopon. Il reprend notamment la conception de l'espace vide tridimensionnel et lui ajoute même ce que JP avait refusé : l'infinitude de l'espace. $A B$ n'est pas atomiste comme le sera Rāzi, mais il réfute les arguments contre la possibilité du vide et même, dans certains endroits, admet son existence.

C'est surtout sa théorie de la connaissance qui semble dès les premières lignes de sa Physique, d'une plus grande liberté que celle de ses prédécesseurs. Par exemple la question du temps, qui empoisonne toutes les descriptions du mouvement depuis l'antiquité : le temps est indispensable à sa description, mais il est lui-même défini par le mouvement. Ce cercle vicieux gênait considérablement Aristote et ses disciples dans leur projet de rationalisation. $\mathrm{AB}$ règle la question dès le $9^{e}$ chapitre en affirmant que l'on peut mener une approche scientifique, en s'appuyant partiellement sur le sens commun (le concept intuitif de temps).

Il reprend l'étude du mouvement avec les mêmes catégories de changement qu'Aristote, il affirme avec lui la nécessité d'un moteur, mais comme JP, ce moteur - qui est autre que le mobile- sera une impulsion que les arabes ont traduit par mayl : mayl qasrī pour le mouvement violent, mayl tab'ì pour le mouvement naturel. En définissant le mayl qasry à la fois comme impulsion transmise par le moteur au mobile et comme résistance au mouvement, Ibn Sīnā - l'aîné d'Abū l-Barakāt, avait introduit une nouveauté : il n'est plus besoin d'évoquer la résistance du milieu pour expliquer la fin du mouvement : il n'y a pas épuisement de mayl (ou d'impulsion) comme le défendait JP et le mouvement peut donc s'envisager dans le vide comme indéfini : une réflexion qui préfigurerait le principe d'inertie si Ibn Sīnā n'ajoutait pas immédiatement, qu'une telle absence de résistance n'a jamais lieu.

$\mathrm{AB}$ reprend et développe cette théorie du mayl : le mayl est aussi bien l'impetus communiqué au projectile que la résistance à la mise en mouvement (ce qu'on appellerait aujourd'hui inertie). 
de lui affecter des puissances puisqu'elles sont ailleurs. L'espace de Jean Philopon préfigure l'espace absolu de Newton : il s'imagine comme une extension tridimensionnelle vide, même s'il ne reste jamais sans corps.

En pensant le mobile séparément de l'espace où il évolue, JP introduit une rupture épistémologique d'une grande fécondité qui ouvre la voie à une mathématisation du mouvement.

\section{III/ Abū l-Barakāt al-Bag̉dādĩ}

Hibat Allah (Nathanaël) 'Ali b. Malkā Abū l-Barakāt al-Bag̉dādī, l'unique de son temps est né entre 1065 et 1075 (soit 30 ans après la mort d'Ibn Sīnā) et meurt vers 1165 (soit 30 ans avant celle d'Ibn Rushd). II aurait grandi à Bagdad élevé parmi les traditionalistes musulmans même s'il semble bien qu'il soit encore juif en 1143 (60 ans). Plusieurs versions des motivations de sa conversion tardive existent mais cela semble peu décisif. Elle freinera peut être la prise en compte de l'héritage du penseur bag̉dādī par les philosophes juifs quoique Crescas (XIVe) reprendra les thèses sur l'infinitude de l'espace, sa tridimensionnalité et l'existence du vide. Du côté musulman, cet héritage sera abondamment commenté par Rāzi (Fakhr ed-Dīn) et en partie par Tūsi dans son commentaire du k. AlIshārāt.

Abū l-Barakāt a écrit un kitāb al-muロatabar en trois volumes : le premier est dédié à la logique, le deuxième aux sciences de la Nature (un commentaire de la physique en 28 chapitres, un traité du ciel, un traité de la $\mathrm{G}$ et $\mathrm{C}$, un traité des météores, et un traité de l'âme) et le troisième est un traité de théologie.

Abū 1-Barakāt al-Bag̉dādī est particulièrement peu connu malgré l'importance de son œuvre, en physique au moins. C'est $S$. Pines qui l'a le plus étudiée : études sur sa théologie, sur la conscience de soi (comparée à celle d'Avicenne) étude sur sa conception du temps (une partie de la physique et surtout dans la Métaphysique). Mais par exemple, la recension de Lettinck (Amsterdam, 91) de toutes les versions de la physique dont le monde arabe disposait ainsi que des commentaires arabes, oublie purement 
distincte d'eux-mêmes.

Philopon ne cherche absolument pas à démontrer l'existence physique du vide, et il faudra attendre encore dix siècles pour que cette question commence à être abordée de façon scientifique. Le rôle du vide de Jean Philopon est donc davantage celui d'outil conceptuel, comme il le dit lui-même à plusieurs reprises, pour penser un espace et un concept de lieu radicalement différent, plutôt que celui d'une réalité physique. Mais il a sérieusement ébranlé l'argument d'Aristote: le vide obstacle au mouvement pour le renverser en «le vide, condition du mouvement ».

\subsection{Les ruptures épistémologiques introduites}

Pour Philopon, l'espace n'a pas de puissance en lui-même.

«Ce n'est pas par désir d'une surface que les choses se déplacent chacune vers leur propre lieu, mais par désir de la position dans l'ordre qui leur a été donné par le Créateur »

La rupture ne saute pas aux yeux d'un scientifique mais pourtant un changement important est à l'œuvre: la cause du mouvement est maintenant dans le mobile et cela justifie à soi seul que l'espace tridimensionnel puisse rester un espace géométrique, vide par définition, et totalement isotrope.

Elle va amener Philopon à conceptualiser y compris pour le mouvement naturel, la notion d'impulsion -rendue par deux mots grecs hormê et rhopê- qui sera nommée plus tard par Buridan, impetus ${ }^{(5)}$. L'introduction de l'impetus consiste à «internaliser » la force responsable du mouvement (en l'absence de principe d'inertie, le mouvement n'est pas imaginable sans force). Ce changement de paradigme, tel qu'il a été argumenté par Philopon en cache d'autres : la physique du ciel par exemple, n'a, a priori, aucune raison d'être différente de celle du monde sublunaire. L'idée est révolutionnaire à l'époque.

Pour Philopon, l'ordre de ce Monde est voulu par un Dieu omniprésent. Il est donc présent jusque dans les corps pour leur indiquer où se trouve leur lieu naturel. Du coup l'espace est « libéré » : nul besoin 


\subsection{Le Commentaire sur le Vide (CV)}

Le centre de cette controverse est la théorie atomiste qui a alimenté un vif débat pendant toute l'antiquité, autour de Démocrite $(-460-390)$, Épicure (-34l-271) et jusqu'à Lucrèce $(-95-55)$ pour ne reprendre qu'au milieu du XVI ${ }^{e}$ siècle (les manuscrits de Lucrèce n'ont été retrouvés qu'au $\mathrm{XV}^{\mathrm{e}}$ siècle). Le vide des atomistes leur permet de construire une science de la Nature d'où seraient exclus le destin, la providence, et leur cortège de peurs, donc la soumission des hommes à la religion. Il n'y a donc rien de plus opposé à la philosophie d'Aristote et la contestation de Jean Philopon ressemble beaucoup, en première lecture, à celle des atomistes.

«Suppose que, quand le mouvement a lieu à travers un milieu corporel comme l'air, les parties de l'air doivent toujours se déplacer sur le côté pour céder au mobile, et la quantité d'air déplacée doit être aussi grande que le mobile, et qu'il n'y a pas d'extension vide qui a reçu le corps déplacé par le mobile - le corps qui a donné de la place pour le mouvement du mobile en la lui cédant- mais l'air déplacé n'a quitté aucune extension : alors pourquoi le déplacement était-il obligé ? "

Pourtant la philosophie des atomistes est bien étrangère à celle du Grammairien, notamment sur la question de l'origine du Monde. Le « Rien ne naît de rien" d'Épicure est inacceptable pour le défenseur de la Création, chrétien, qu'est Jean Philopon. Ce dernier a besoin de débarrasser l'espace des "principes» et des causes dont il est encombré car c'est au Créateur qu'il veut donner ce pouvoir d'ordonner le Monde. Le concept de vide qu'il va élaborer n'est donc pas celui de Démocrite ou d'Épicure. Ce n'est pas un vide «étant» mais un vide «conceptuel» qui existe comme extension tridimensionnelle car il n'est jamais sans corps. C'est le sens d'une très longue démonstration de JP dans son $\mathrm{CV}$ à propos des clepsydres.

$\mathrm{Ce}$ qui est vide, ou plutôt risque de le devenir, assène le Grammairien, c'est l'extension tridimensionnelle et rien d'autre. Une extension que le Créateur a adjoint aux corps, en plus d'eux-mêmes donc 
sera redécouvert, qu'après la traduction de son traité de l'âme par Guillaume de Moerbeke (XIIIe siècle).

Moins de cinquante ans après la disparition de Jean Philopon, l'Égypte est conquise par les musulmans. C'est ce monde musulman qui va, le premier, hériter de l'œuvre de Philopon, œuvre que Ibn Sînâ, Abu l'Barakat, et Ion Bājja reprendront et commenteront.

\subsection{Le Commentaire sur le lieu (CL)}

Le système du monde aristotélicien est difficile à attaquer de façon parcellaire et Jean Philopon le sait. Il ne veut d'ailleurs pas l'écrouler mais le rendre plus cohérent.

L'espace tridimensionnel, vide de tout corps par définition, qu'il propose de substituer à « la première enveloppe immobile » n'est pas du tout un objet inconnu de la philosophie grecque: c'est l'espace géométrique, et pour une bonne part la X $\omega \rho \alpha$, de Platon. Le problème est de l'introduire dans une Physique dont il partage l'objectif avec Aristote : étudier la nature, rendre compte de ses mouvements, et notamment du mouvement local, à partir de données tangibles.

Jean Philopon va donc détacher le lieu des corps qui l'occupent en l'imaginant comme l'espace de Platon, vide par définition et par nature immuable, puisque non corporel (et que seuls les corps sont soumis au changement). Ce lieu n'est pas un objet strictement mathématique qui, échapperait comme celui du Timée «aux sens par lesquels nous percevons les êtres changeants et corruptibles ». En bon aristotélicien ${ }^{(4)}$, JP veut lui donner une «réalité physique » dirions-nous aujourd'hui qui est celle de l'extension tridimensionnelle. JP va aussi combattre la définition d'Aristote en introduisant une réflexion sur la mesure du corps (sous entendu celle de son volume) et sur la dimension (espace/surface) ce qui confirme bien l'idée que la topologie est au centre du problème. 


\section{II/ Jean Philopon, l'alexandrin}

Jean Philopon ${ }^{(3)}$ a sans doute vécu de 490 à 575 environ, soit plus de quatre vingt ans. Il est un sujet de l'empire romain d'Orient (l'Empire byzantin), et vit pour l'essentiel sous le règne de Justinien $1^{\text {er }}$. Il est grec par sa culture, égyptien par son lieu de naissance, et chrétien par son baptême.

Il a vécu à Alexandrie dans le diocèse d'Égypte où il a été l'élève d'Ammonius, le fils d'Hermeias qui dirigeait lui-même l'école d'Alexandrie au début du $V^{e}$ siècle. L'institution d'une authentique école de philosophie à Alexandrie date de cette époque.

L'école d'Alexandrie est marquée par une entente entre chrétiens et païens qui contraste avec la situation à Athènes où Proclus avait attaqué violemment les chrétiens mettant en cause leur croyance en l'existence d'un commencement du Monde. Au contraire Ammonius et ses trois élèves Philopon, Elias et Simplicius enseignent une interprétation de la pensée d'Aristote intégrant la reconnaissance d'un Dieu créateur du monde physique. C'est ce qui sauvera l'école d'Alexandrie alors que celle d'Athènes sera fermée en 529.

JP a affirmé sa conviction en l'existence d'un commencement du Monde dans son commentaire de la physique d'Aristote (qui date de 517). Certes les grecs païens croyaient que l'organisation du monde et de la matière avait un début. Mais pas la matière elle-même puisqu'ils refusaient l'idée d'une création ex-nihilo.

C'est comme philosophe, physicien et logicien que Jean Philopon a mené ses travaux contre la Physique d'Aristote. Il s'est penché par la suite sur les questions de doctrine religieuse: le Christ, la Trinité et la résurrection. Tout laisse penser que sur la fin de son existence au moins, ses rapports avec les autorités chrétiennes se sont fortement détériorés. En tout cas en 680 , soit cent ans après sa mort, il fut frappé d'anathème pour sa mise en cause de l'existence de la Trinité. Ceci explique largement pourquoi Philopon a été totalement ignoré par le Moyen Age latin et ne 
ou du mouvement des corps. Pour lui, il ne faut pas confondre le lieu et le vase : en effet le vase peut être déplacé, alors que le lieu lui, doit être immobile par nature. Le lieu d'un bateau amarré au bord d'un fleuve n'est donc pas l'eau qui l'entoure puisque celle-ci est en mouvement, mais le lit du fleuve tout entier car il est immobile. Cette définition ne peut pas être comprise sans la relier à sa conception de l'espace : il est totalement plein, fini, et bomé par la sphère des fixes. Définir le lieu comme une extension tridimensionnelle (comme le faisait Platon) pose la question de la pénétrabilité des corps. La tridimensionnalité est pour lui une propriété définissante des corps et il est donc impossible de l'affecter au vide. Aristote défend donc l'idée que le lieu d'un corps «n'est pas autre chose que la partie, immédiatement contiguë à ce corps, du milieu qui l'environne »:

Cette définition pose problème pour le huitième orbe qui est le lieu de l'Univers pour Aristote. La question est épineuse car il est en mouvement autour de l'axe du Monde. Mais alors qu'y a-t-il après ? Rien ? Pas même le vide? L'infini ? C'est impensable! La seule solution aurait été d'imaginer une sphère céleste immobile. Or celle-ci, serait donc acte pur et donc « intelligence séparée » et non pas corps : elle ne pourrait pas loger l'Univers. Aristote s'en était sorti (plutôt mal que bien) en précisant que les diverses parties du 8eme orbe sont un lieu d'une certaine manière, un lieu par accident.

Le décor est planté : pour expliquer le mouvement des corps, qu'il soit violent ou naturel, il faut statuer sur l'existence du vide et sur la notion de lieu, aujourd'hui on dirait d'espace. Ce sont ces notions qui vont animer plusieurs commentateurs : Jean Philopon (Alexandrie $m$ 575) le plus ancien de tous, Avicenne (m 1037) le plus oriental géographiquement (persan de Boukhara), et Abū l-Barakāt al-Bağdādì (m 1165). Il faut donc faire un détour par ces derniers. 
mouvement que connaisse le monde supra-lunaire est le mouvement circulaire, mouvement selon le lieu. Ce monde est constitué d'un seul élément : l'éther.

Le monde sublunaire est constitué lui des quatre éléments (la terre au centre, et concentriquement l'eau, l'air et le feu.) et connaît les quatre types de changement d'Aristote : la naissance et la disparition des substances (la génération et la corruption), le changement qualitatif, le changement quantitatif (la raréfaction et la densification ou la croissance des vivants) et enfin le mouvement local. Si un morceau d'élément est déplacé de son lieu naturel, le mouvement qui l'affecte alors est un mouvement violent; lorsqu'il retoume à son lieu naturel, son mouvement est alors un mouvement naturel. Si je lance une pierre en l'air, je lui impose un mouvement violent. Dans tout mouvement violent, il y a, selon Aristote, un moteur et un mobile qui restent en contact l'un avec l'autre. Dans le mouvement d'un projectile, le moteur n'est plus en contact avec le mobile, il en est séparé. Comment expliquer alors que le projectile continue à se mouvoir? Pour Aristote la cause doit coexister avec son effet. Le rôle moteur sera donc donné au milieu (à l'espace), l'air ou l'eau. Le lanceur transmet à la première portion d'air à la fois un mouvement et une capacité motrice. La capacité motrice dure plus longtemps que le mouvement, de sorte que la portion d'air motrice peut à son tour transmettre à la portion suivante à la fois un mouvement et une capacité motrice plus faible que la première et ainsi de suite de proche en proche, jusqu'au moment où durée du mouvement et durée d'action de la portion d'air considérée coïncident. Le mouvement s'arrête alors.

Enfin pour définir le mouvement local, Aristote est obligé de définir le lieu en cohérence avec sa cosmologie; cette définition sera le talon d'Achylle de son système du monde que ne manqueront pas d'exploiter les deux commentateurs que nous allons évoquer. Aristote affirme que le lieu est la frontière commune appartenant à la fois au corps contenu et à l'objet contenant. Il veut que le lieu permette de «juger » de l'immobilité 
inventeurs de l'astrolabe, les systèmes d'irrigation et les machines hydrauliques d'al-Jazari, les norias de Dama, les recettes alchimiques d'alJāber....).

En résumé la science comme théorie serait grecque et la science comme démarche expérimentale, celle que nous appelons sciences physiques, serait européenne, née au XVIIème siècle... au monde arabomusulman on ne reconnaîtrait que le mérite d'avoir traduit et bricolé... Cette conception est pour le moins discutable.

La présentation qui va suivre s'inspire des travaux de Shlomo Pinès ${ }^{(1)}$, qui est le premier à avoir vraiment étudié la physique d'Abū lBarakāt al-Bag̉ā̄ī, objet de mes recherches, et d'Ahmed Hasnaoui ${ }^{(2)}$ qui a produit de nombreux travaux sur la physique du Shifā' d'Ibn Sīnā et sur la transmission de la philosophie du grec à l'arabe.

\section{I/La physique du mouvement des projectiles chez Aristote.}

Développée essentiellement dans les livres IV et VIII de la Physique, un de ses objets d'étude est le mouvement d'une pierre jetée en l'air et qui retombe : le mouvement ascendant « contre-nature » et dont la «cause » est le lanceur s'appelle mouvement forcé ou mouvement violent; le mouvement descendant étant appelé lui, mouvement naturel. Le repos intermédiaire situé en haut du mouvement et qui a fait coulé beaucoup d'encre chez les commentateurs latins étant nommé le quies media (repos intermédiaire).

Aristote sépare deux physiques, celle du ciel, le monde supralunaire (au dessus de la sphère de la Lune) et celle du monde sublunaire (le notre). Il a construit un Monde entièrement hiérarchisé et d'une immense cohérence. Il cherche une explication matérielle à chaque phénomène, qui tranche avec les croyances et mythologies de son temps.

Le monde d'Aristote est sphérique, son centre est celui de la Terre (appelé centre du Monde ou centre des graves) et géostatique. Le seul type de 


\title{
La Physique du mouvement, de l'antiquité tardive de culture grecque au Moyen Age arabe.
}

\author{
Sylvie Nony \\ Professeur agrégée de Physique \\ Doctorante d̀ Paris VII, unité d'accueil du CHSPAM \\ Centre d'Histoire des Sciences et des Philosophies Arabes et Médiévales \\ Recherches menées da la bibliothèque de l'Institut des Études Dominicaines du Caire.
}

L'histoire des sciences de « la physique du mouvement », telle qu'elle est enseignée aujourd'hui du secondaire à l'Université, présente un gouffre historique. Il n'y aurait eu que deux étapes importantes: la théorie d'Aristote exigeant «que tout mobile soit mû", et celle de Galilée élaborant le principe d'inertie. Il s'agit d'une véritable reconstruction de l'histoire dont un des buts est de situer l'an zéro de la science moderne au XVIIème siècle en plein cœur de l'Europe.

Laisser penser que pendant 20 siècles, l'humanité n'a rien produit d'autre que du commentaire ronronnant d'Aristote est assez étonnant. De plus, la conception du moyen âge qui accompagne ces affirmations est particulièrement contestable. Le moyen âge méditerranéen n'y est présenté - à la suite de l'antiquité tardive- que comme une période de fermeture souvent imaginée dans le prolongement d'une fin d'empire et d'une décadence.

Quant à l'apport du moyen âge oriental, et notamment du monde arabo-musulman, il est complètement nié. Jusqu'à la deuxième moitié du $\mathrm{XXe}$, les analyses les plus érudites (Duhem) ont présenté pour l'essentiel la science arabe comme un conservatoire (attentif) de la science grecque d'une part, et/ou comme un apport essentiellement technologique (les 